\title{
Three Delays and LAMA (Left Against Medical Advise): Verbal Autopsy of Still Birth from North India
}

\author{
Arun K Aggarwal* and Dhritiman Das \\ School of Public Health, Postgraduate Institute of Medical Education and Research, India
}

Submission: December 15, 2016 ; Published: January 09, 2017

*Corresponding author: Arun K Aggarwal, School of Public Health, Postgraduate Institute of Medical Education And Research, Chandigarh, India, Email: aggak63@gmail.com

\begin{abstract}
Stillbirths are huge public health burden in the developing countries. There is gross lack of understanding about the factors contributing to the Stillbirths. Three delay models are well documented to describe delays at family level, transport level and facility level based on Verbal Autopsies. There is a general tendency to put all blames on the family for any adverse outcome. High number of "LAMA" (Left against Medical Advise) cases are often attributed to poor treatment seeking behavior of the families. However, this may also be due to poor quality of care being delivered to the families.

We did community based verbal autopsy for stillbirth review and extended the case to get more information from hospital based enquiries. The mother felt in late evening that foetus has stopped moving in her womb, but delayed seeking consultation till next morning.. At the primary health centre time was wasted due to non availability of health staff, and in getting the investigations done. Non-availability of appropriate transport contributed further to the risk. Lastly, the hospital staff did not attend to the case for 30 hours. After long wait at the hospital, the poor family decided to shift to the private hospital. This could have led to maternal death that was averted due to alertness of family. However, Govt. hospital labeled this case as "LAMA" (Left against Medical Advise). We recommend toput in place strong accountability frameworks in the hospitals to avert such situations.
\end{abstract}

Keywords: Still birth; Verbal autopsy; LAMA; Community; India

Abbreviations: LAMA: Left Against Medical Advise

\section{Introduction}

Patients get admitted to the hospitals/ health facilities to seek treatment/ resolution of their problems. However, it has been reported in literature that many patients leave the hospitals before their treatment gets completed. Such patients are generally considered to be "Discharged against medical advice" or "Left against Medical Advise" (LAMA) in the medical terms. This problem of patient discharges against medical advise, has emerged as a pervasive problem in general hospitals. Terminology puts the blame on the patients. It denotes that there is problem in the health seeking behavior of the patients. However, literature review shows that a patient leaves the hospital when his/her expectations are not fulfilled or they get threatened due to any social or economical reasons. Patients may withdraw consent from continuing treatment, against medical advice, for a number of individualized reasons that may range from personal, family, or financial issues; conflicts with staff; dissatisfaction with hospital care, environment, or treatment interventions; and misunderstandings based on underlying medical, cognitive, and psychiatric issues) [1-3].

There are some cases where refusal for treatment is on account of the organic confusions on the part of the patients [46]. However, adults with sound mind if are withdrawing consent or are leaving against medical advice, then it may denote serious problems with the health systems.

LAMA cases put an important challenge to any responsive health care organization.. There are experiences that with better understanding of patients' issues and better communication skills such patients can be managed with greater success. However, many health organizations hide their irresponsible behavior under the mask of "LAMA", so that blame is put on the 
patients itself. In fact, it is well documented in literature that majority of the LAMA patients and their care givers are either generally uninformed or less informed of the entailing side effects and outcomes of their decision to leave against medical advice [7].

We are reporting here one case study to highlight this problem and discuss the case to draw lessons and viewpoint for health systems improvements.

\section{The Context}

This north Indian state is one of the progressive states in India in terms of economic and infrastructural development; however, it still lags behind tremendously in the area of medical and social development. The current Infant Mortality Rate (IMR) of state is $42 / 1000$ live births and it is still yet to catch up a long way to reach its Millenium Development Goal (MDG) target of 30 per 1000 live births. With the deadline of achieving the MDG target nearing in time; the state health department under the leadership of National Health Mission officers; has been implementing various programs to check and bring down overall IMR in the state. The Infant Death Review (IDR) program was formally implemented in 10 high priority districts of state since 2013 with the intention of tackling infant deaths, stillbirths and maternal deaths. In regard to this program, as an external research body; this team had come across one case of stillbirth that was reviewed qualitatively.

The following history is based on the verbal autopsy recorded from the family members (parents) of the deceased intrauterine death: Mrs "X" was pregnant for the second time, with a healthy first born baby. She was fully compliant woman with respect to early antenatal registration and adequate antenatal checkups with the routine health system. She had also consumed tablets of iron and folic acid as advised; considering anemia to be a highly prevalent health problem in the areas of her dwelling with poor compliance to its management in general.

At $8^{\text {th }}$ month of gestation, this antenatal mother (Mrs. $\mathrm{X}$ ) underwent ultrasound examination and was detected to be having Breech presentation of the foetus. Health service providers assured her that there was no need to worry, as this position could autocorrect itself. At 9 th month of gestation, one evening, mother felt that the foetus had stopped moving in the womb. She waited overnight anticipating the baby's movement, and next morning visited the nearest block level health facility with her spouse on their personal two-wheeler. However, they had to wait for health staff for more than two hours, as no one was available at the primary health centre (PHC) to attend to her concern. That particular day was a Sunday (holiday). (A Primary Health Centre or PHC, is supposed to be running all 24 hours for 7 days a week and even during holidays, under the supervision of a doctor and nurse). Later, a Staff nurse arrived and checked the mother; and she realized that the Foetal Heart Sounds (FHS) of the unborn baby was either scanty or not audible. The PHC nurse asked them to get an ultrasound done from a private service provider, instead of referring to general hospital of the district where out of pocket expense would have been saved. This decision made by the staff nurse was autonomous and without any consultation with the on call duty Medical Officer in charge of the PHC. Thereafter, the couple went to the suggested private clinic on their own two wheeler, traversing a road terrain that was pebbly and difficult. Ultrasound report done at the private clinic indicated that the foetus was dead. The family came back to the PHC nurse for consultation and now the mother was referred to general hospital for delivery. This time, the Staff Nurse in question had called for free government ambulatory service to transport the patient to the higher level hospital; however, the ambulance never came and the couple had to continue their journey via the same two wheeler. At the general hospital (District level higher center) the staff nurse on duty admitted the mother after 4 hours of struggle. She was not willing to admit. Lot of pressure had to be built on her from district and state for the admission to happen. However, even after admission she was not attended to adequately. The duty doctors on call also refused to attend the patient in the hospital. She remained admitted without proper treatment for more than 30 hours. She was having pain but there was no hearing of her problem. The couple insisted on getting delivered at the government facility because the baby was already dead and since they were from a socioeconomically weak background who couldn't afford treatment at private hospital for a lost cause. At last, the dissatisfied family took the mother to private hospital where she got delivered in half an hour.

General hospital records file mentions "LAMA"

\section{Discussion}

Three-Delays-Model is often used for health system improvement in the field of maternal and child health care. According to this model, the three levels of delays leading to infant/maternal death are as follows: Delay 1: Delay in decision making to seek care (Family Level or lack of awareness), Delay 2: Delay in reaching health care facility (or transportation delay), Delay 3: Delay in receiving adequate care at tertiary level centers (also includes delay in referral from one facility to another) All three delays occurred in this case. The history denotes that despite being aware about the routine antenatal care, Mrs. "X" lost her baby in the womb, because she delayed her first decision for seeking consultation after she could not feel the foetal movements overnight. Moreover, her spouse or family members did not necessarily feel the need to seek immediate treatment the moment this problem started and instead waited an entire night before seeking care. This may be due to poor quality of counseling given during antenatal period to mothers on delivery preparedness during their $3^{\text {rd }}$ or $4^{\text {th }}$ antenatal visits and thus required awareness was not created regarding possible complications. Had the mother and family members known of the dangers signs of pregnancy and the importance of prompt treatment, it is anticipated that they would have visited the health facility immediately. 
Secondly, it was evident from the history that the Mrs. " $\mathrm{X}$ " and her spouse never received the entitled free government ambulatory services throughout their attempts to seek care at various levels of the health care system. They had to instead travel on a two-wheeler riding over a terrain that was reportedly not an easy to ride area. This possible disturbing ride could have further aggravated the conditions of her foetus; considering it was already having a faint Foeatal Heart Sound (FHS). Moreover, this case indicates a lack of proper communication between health facilities and the ambulance control room staff which resulted in unavailability of ambulance for this concerned case.

Thirdly, from the perspective of delay in prompt and appropriate referral; it can be easily pointed out that initial management at the PHC level had several underlying loopholes which could have directly/indirectly contributed to the resultant intrauterine death. According to the recorded history, there was no staff available to attend Mrs. "X" at the PHC for 2 hours for a health facility that is expected to function round the clock and throughout the week. Later when the staff nurse arrived, even after knowing that FHS of the foetus was scanty; she instead chose to send the patient in some private clinic for an ultrasound instead of directly referring the case to a higher government facility via free government ambulance. Moreover, the staff nurse did not bother to consult the supervising medical officer in charge of the PHC before making decision about this particular case. Had she been punctual and made decisions based on consultation with superiors, delay 3 could have been partially avoided.

However, apathy of the health system unfolds further when she reaches district level general hospital on a holiday, with a dead foetus in womb, and was not taken care of. The couple insisted on getting delivered at the government facility because the baby was already dead and since they were from a socioeconomically weak background who couldn't afford treatment at private hospital for a lost cause. However, no one at the district level hospital attended to their concerns for more than 30 hours. Everyone including staff nurses, duty doctors and higher officials chose to shirk away from their responsibility. This undelivered intrauterine death could have also led to a possible maternal death. With the history quoted in the verbal autopsy, this is a clear case of dereliction of duties and the patient was indirectly contemplated into seeking care elsewhere. However, labeling such cases as "LAMA" has serious program implications.

\section{Conclusion}

Health facilities should ensure that clients are able to recognize the seriousness of the complications and take timely decisions to seek consultation. Accountability frameworks should be put in place to avoid delays in providing health care/ treatment. All "LAMA" cases should be put under active surveillance to identify potential gaps in the provision of hospital care.

\section{Acknowledgements}

This case study was generated from the project funded by National Health Mission of a State. Dr. Himani Anand, project data analyst helped in improving the manuscript, Dr Rakesh Gupta, MD (NHM) funded the project, Dr Suresh Dalpath ( State deputy director child health) liased with the districts for supporting the project.

\section{References}

1. Albert HD, Kornfeld DS (1973) The threat to sign out against medical advice. Ann Intern Med 79(6): 888-891.

2. Long JP, Marin A (1982) Profile of patients signing against medical advice. J Fam Pract 15(3): 551-556.

3. Onukwugha E, Saunders E, Mullins CD, Pradel FG, Zuckerman M et al. (2010) Reasons for discharges against medical advice: a qualitative study. Qual Saf Health Care 19(5): 420-424.

4. Brody B (2009) Who has capacity? N Engl J Med 361(3): 232-233.

5. Jones JW, McCullough LB (2008) The shifting sands of senility: canceled consent. J Vasc Surg 47(1): 237-238.

6. Jones JW, McCullough LB, Richman BW (2007) A comprehensive primer of surgical informed consent. Surg Clin North Am 87(4): 903-918.

7. Noohi K, Komsari S, Nakhaee N, Yazdi Feyzabadi V (2013) Reasons for Discharge against Medical Advice: A Case Study of Emergency Departments in Iran. Int J Health Policy Manag 1(2): 137-142.

\section{Your next submission with Juniper Publishers} will reach you the below assets

- Quality Editorial service

- Swift Peer Review

- Reprints availability

- E-prints Service

- Manuscript Podcast for convenient understanding

- Global attainment for your research

- Manuscript accessibility in different formats

( Pdf, E-pub, Full Text, Audio)

- Unceasing customer service

Track the below URL for one-step submission https://juniperpublishers.com/online-submission.php 